Supporting Information for:

\title{
Light-activated peptide-based materials for sutureless wound closure
}

Christopher D. McTiernan, ${ }^{1}$ David C. Cortes,${ }^{2}$ Caitlin Lazurko, ${ }^{1}$ Selya Amrani, ${ }^{1}$ Roberto Rosales-Rojas, ${ }^{3}$ Matias Zuñiga-Bustos, ${ }^{3}$ Veronika Sedlakova, ${ }^{1}$ Horacio Poblete, ${ }^{3}$ Kevin Stamplecoskie, ${ }^{4}$ Erik J. Suuronen ${ }^{1}{ }^{1}$ and Emilio I. Alarcon ${ }^{* 1,5}$

${ }^{1}$ BEaTS Research, Division of Cardiac Surgery, University of Ottawa Heart Institute, Ottawa, Ontario, Canada, K1Y 4W7.

${ }^{2}$ Biomedical Mechanical Engineering, University of Ottawa, Ontario, Canada, K1N 6N5.

${ }^{3}$ Center for Bioinformatics and Molecular Simulations, Facultad de Ingeniería, Universidad de Talca, Campus Lircay S/N, Talca, Chile, 3460000; Millennium Nucleus of Ion ChannelsAssociated Diseases (MiNICAD), Universidad de Talca, Chile, 3460000.

${ }^{4}$ Queen's University, Chemistry, Chernoff Hall Rm 505/435 90 Bader Lane, Kingston, Ontario, Canada, K7L 3N6

${ }^{5}$ Department of Biochemistry, Microbiology, and Immunology, Faculty of Medicine, University of Ottawa, Ottawa, Ontario, Canada, K1H 8M5. 


\section{Table of Contents}

S1. Femtosecond Transient Absorbance (pump/probe) spectroscopy (fsTAS) of S-3 Thin Films

S2. Pore size analysis of RB-CLPI hydrogels via Cryo-SEM

S3. Denaturation temperature of optimized formulations (Differential Scanning S-4 Calorimetry)

S4. Protein expression profile within Sutured and CLP treated dorsal wounds 7 S-4 days post-treatment

S5. Schematic and Key Components of Irradiation System

S-5 


\section{S1. Femtosecond transient absorbance (pump/probe) spectroscopy (fsTAS) of thin films}
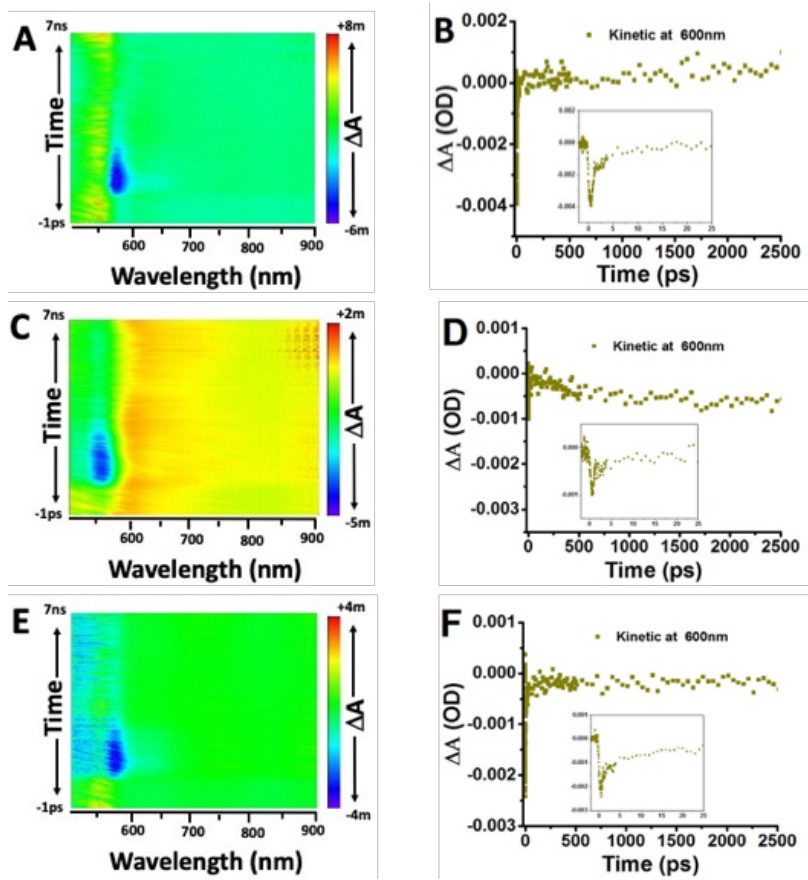

Figure S1.3D transient absorption surface (colour is the magnitude of $\triangle \mathrm{A}$ as indicated) for CLL films containing RB alone (A), RB with CLPI (C), and RB with CLPII (E). Figures (B), (D), and (F) are the corresponding kinetic profiles of the $\Delta \mathrm{A}$ vs time measured at $600 \mathrm{~nm}$, which was selected because it illustrates both the rapid relaxation of $\mathrm{RB}$, and the growth in a reaction product formed from reaction with the triplet excited state of RB in (D).

\section{S2. Pore Size Analysis of RB-CLPI Hydrogels via Cryo-SEM}
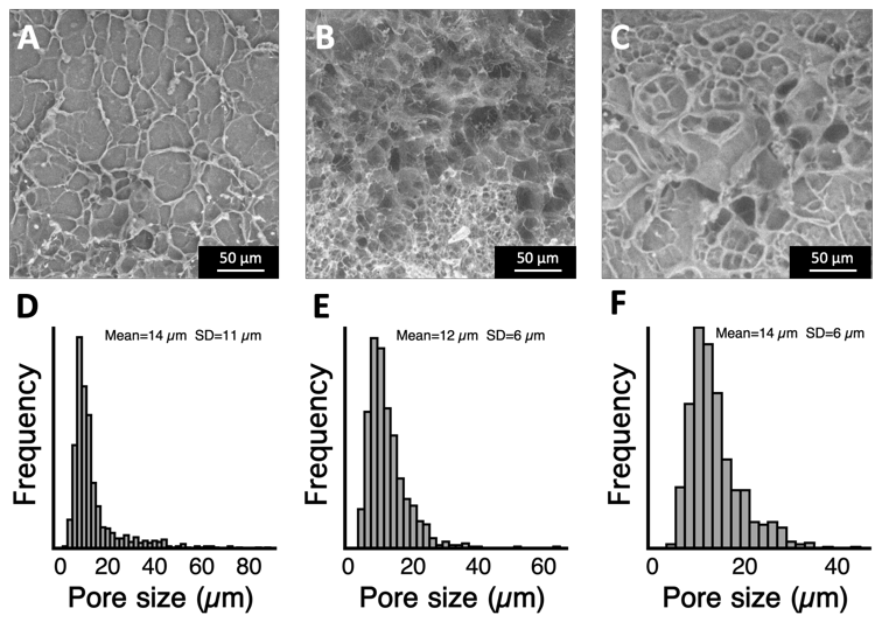

Figure S2. Cryo-SEM images of CLPI-PEG-Ac hydrogels obtained after (A) $10 \mathrm{~min}$, (B) $15 \mathrm{~min}$, and (C) $30 \mathrm{~min}$ of irradiation with $12 \mathrm{mg}$ 20K 8-Arm-PEG-Ac. (D)-(F) Corresponding pore size histograms for 10, 15, $30 \mathrm{~min}$ irradiation. 


\section{S3. Denaturation Temperature of Optimized Formulations (Differential Scanning Calorimetry)}

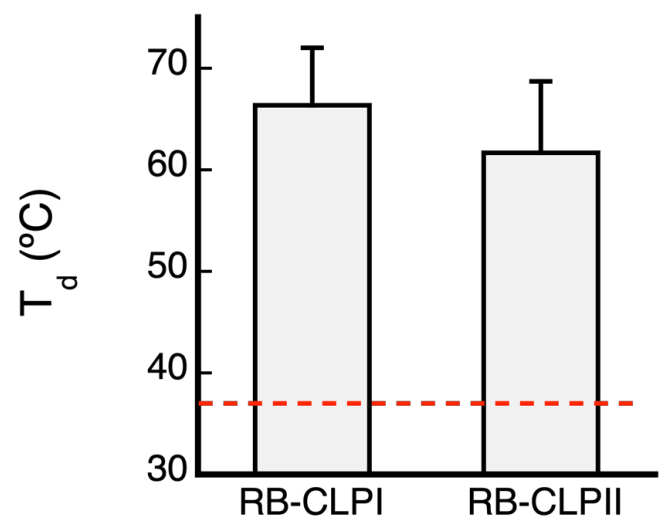

Figure S3. Denaturation temperature $\left(\mathrm{T}_{\mathrm{d}}\right)$ of the optimized RB-CLPI and RB-CLPII hydrogels. Formulations consisted of $12 \mathrm{mg} 20 \mathrm{~K}$ 8-Arm Polyethylene Glycol Acrylate [hexaglycerol core] (8A-PEG-Ac), $10 \mu \mathrm{L}$ of $2.5 \mathrm{mM}$ Rose Bengal, $50 \mu \mathrm{L}$ of $0.34 \%$ PLL, and $20 \mathrm{mg}$ of either $30 \% \mathrm{w} / \mathrm{w}$ CLPI-PEG-Ac or CLPII-PEG-Ac irradiated for $15 \mathrm{~min}$. Values in plots correspond to the average of $n=3$ samples.

\section{S4. Protein Expression Profile within Sutured and CLP Treated Dorsal Wounds at 7 Days} Post-treatment
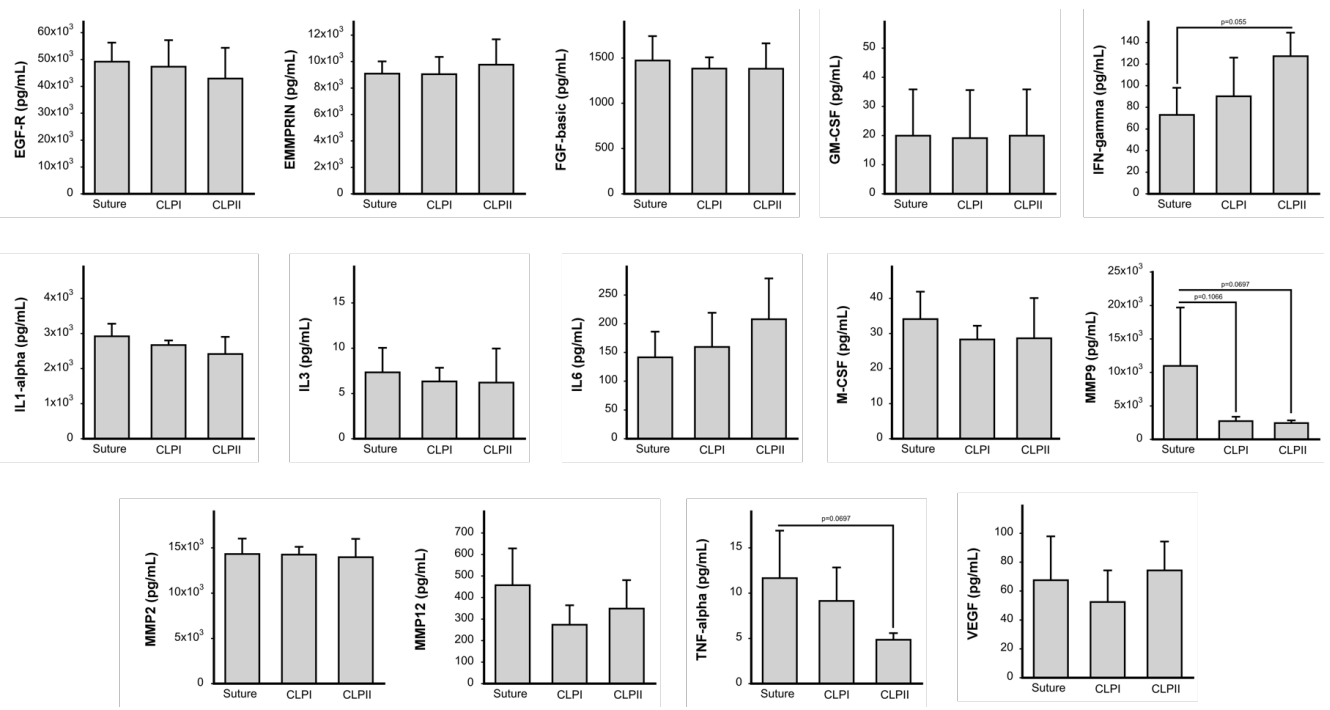

Figure S4. Luminex multiplex assay showing protein concentrations in the skin of Suture, RB-CLPI, and RB-CLPII treated wounds at 7 days post-treatment. Values correspond to the average of $n>3$ samples. Error bars correspond to standard deviation of measurement. $P$-values were calculated using One Way ANOVA test followed by Tukey's post hoc test. 


\section{S5. Schematic and Key Components of Irradiation System}

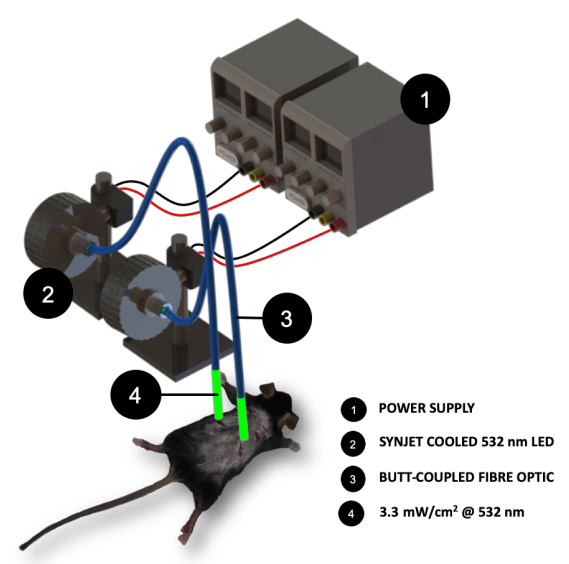

Figure S5. The crosslinking of the gels was performed using a custom-made illumination system coupled to a fibre optic cable. The distance between the LED/fibre optic cable $\left(\lambda_{\mathrm{en}}=523 \mathrm{~nm}\right)$ and the gel was approx. $5.5 \mathrm{~mm}$ giving an irradiance of $3.3 \mathrm{~mW} / \mathrm{cm}^{2}$.

The experimental setup was built using a $523 \mathrm{~nm}$ LEDs purchased from Mouser Electronics (LED Engin - LZ4-40G100) mounted onto drilled and tapped SYNJET cooled heatsinks (DigiKey) using thermal paste and matching bolts. The mounted LEDs were then fixed to an optical breadboard and supplied by a DC power supply with the current and voltage set to 0.7A and 14.0V, respectively. Light from the LED system was brought to the operating table via Fibre Optic cable (THORLABS, FP1500URT) that had been cut to 45 inches of length, polished, and butt-coupled to the dome of the LED through its attachment to a repurposed mechanical microscope slide scanner, which when fixed to the optical breadboard allowed for fine adjustment of the LED-Fibre alignment. 